\title{
Research on the Acoustic Insulation Properties of PVC
}

\author{
Liping Zhang ${ }^{1, a}$ \\ ${ }^{1}$ School of mechanical and electrical engineering, Foshan University, Foshan, 528000, China \\ aemail: Ipzhangok@163.com
}

Keywords: PVC; $\mathrm{CaCO}_{3}$; Acoustic Insulation; High-frequency Noise; Areal Density

\begin{abstract}
PVC is an acoustic insulation material in the air conditioner with low cost. In order to study its acoustic insulation experiments were done for the white noise and compressor noise. Acoustic insulation and the noise reduction spectrum of PVC were obtained which show that PVC can effectively reduce the high-frequency noise and the acoustic insulation of PVC increases with the areal density increasing, however, $\mathrm{CaCO}_{3}$ content has little effect on acoustic insulation of PVC.
\end{abstract}

\section{Introduction}

With the development of the industry, noise pollution has become a worldwide problem which influence people's health and work efficiency. As a necessary electrical apparatus the air conditioner will produce noise during working so noise reduction must be done during using. Now there are two methods of noise reduction, that is, sound absorption and sound insulation. Acoustic insulation is to isolate the noise source and the surrounding environment and then the noise radiation can be reduced. PVC can be is used to insulate the compressor noise instead of Neoprene with lower cost.

More and more scholars have study on acoustic insulation of PVC now. Leli Cheng pointed out that the addition of flame retardants composite material does not influence the acoustic insulation, but it is able to improve the flame-retardant and smoke suppression properties of PVC [1]. Tianbing Yang study the acoustic insulation of honeycomb weave fabric/PVC composite material. The results show that repetitions of weave have great influence on the acoustic insulation effect of honeycomb weave fabric [2]. Hui Liu found that fillers such as DOP, CPE and $\mathrm{BaSO}_{4}$ make the processing performance and mechanical properties of PVC poor but have little effect on the acoustic insulation [3]. Zhongcheng Fang found that PVC had good effect on noise of the refrigerator by test [4]. Handong Xue pointed out PVC with sound-absorbing material can more effectively reduce the noise of the air conditioning than only absorption material, but the cost and installation must be considered in application [5]. Lei Huang found that sound insulation cover made from PVC/PET fiber can effectively reduce the noise of the compressor by simulation and tests [6]. Our team study on the PVC filled with $\mathrm{CaCO}_{3}$ and found that PVC is an insulation materials with high performance-price ratio in air conditioning. When $\mathrm{CaCO}_{3}$ content is lower than $60 \%$ its sound insulation, tear strength and heat resistance are higher than Neoprene [7]. Based on this, the paper focus on the characteristic of the acoustic insulation of PVC filled with $\mathrm{CaCO}_{3}$, which can provide further theoretical basis for the application of PVC.

\section{Design of the test methods}

PVC can be mainly used to deaden the compressor noise. In order to study its characteristics of sound insulation, tests were done in lab. The unit were tested to avoid the effect of the compressor and assembly error. The sound insulation test plan is shown in Fig.1. The recording of the compressor working at $83 \mathrm{~Hz}$ and white noise are sources placed in asemi-anechoic room in test. Through the acoustic pipe noise sources transmit into the sound chamber. Noise sources were reflected and refracted by the acoustic insulation rubber and reached to the pickup. Sound-absorbing material was added to the inside of the acoustic pipe to avoid pipe resonance. In the experiment the 
acoustic insulation rubbers were made from PVC and Neoprene which sealed the end of the acoustic pipe in the sound chamber with shockproof. The distance is $100 \mathrm{~mm}$ between the pickup and insulation rubbers. The thickness of insulation rubbers is respectively $1 \mathrm{~mm}$ and $2 \mathrm{~mm}$ and the content of $\mathrm{CaCO}_{3}$ in PVC is respectively 45\%, 50\%, 55\%and60\%. The size of the rubbers is 150 $\mathrm{mm} \times 150 \mathrm{~mm}$.

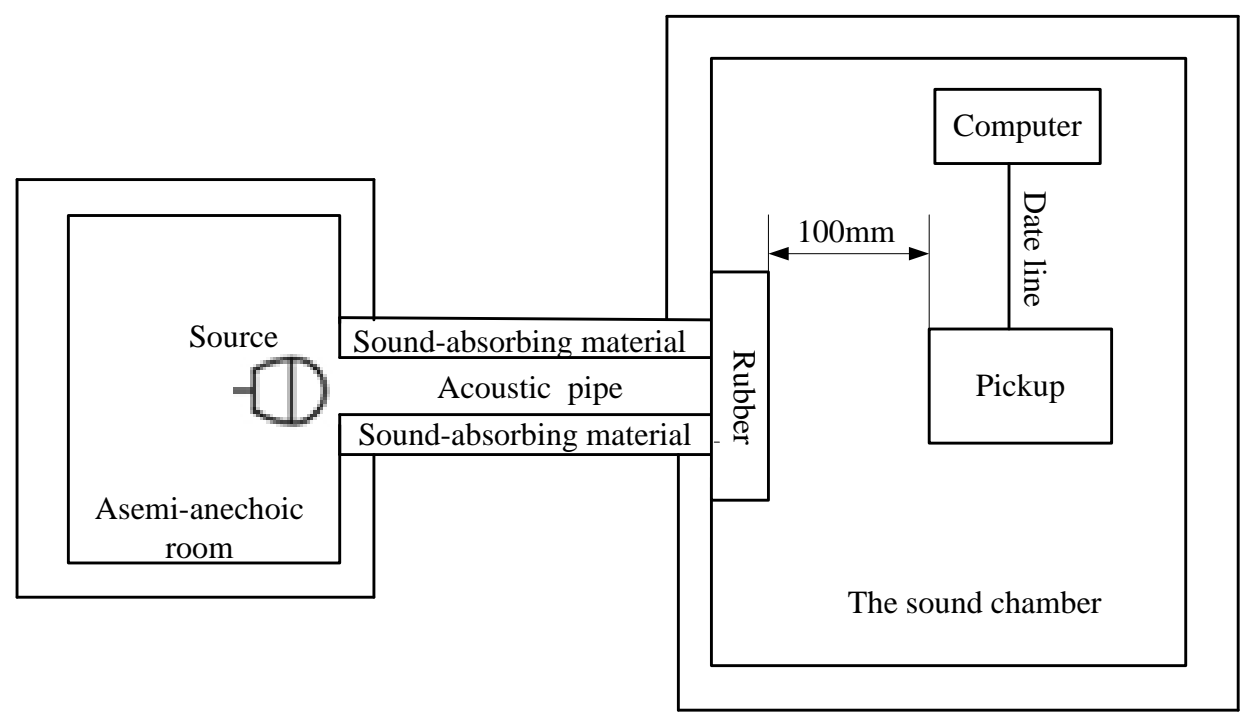

Fig.1. Schematic diagram of the test system

\section{Noise reduction curves of PVC}

Noise spectrum curves of original noise and noise after insulated by PVC are compared in Fig.2 in which the thickness of PVC is respectively $1 \mathrm{~mm}$ and $2 \mathrm{~mm}$ with $\mathrm{CaCO}_{3}$ content of $50 \%$.

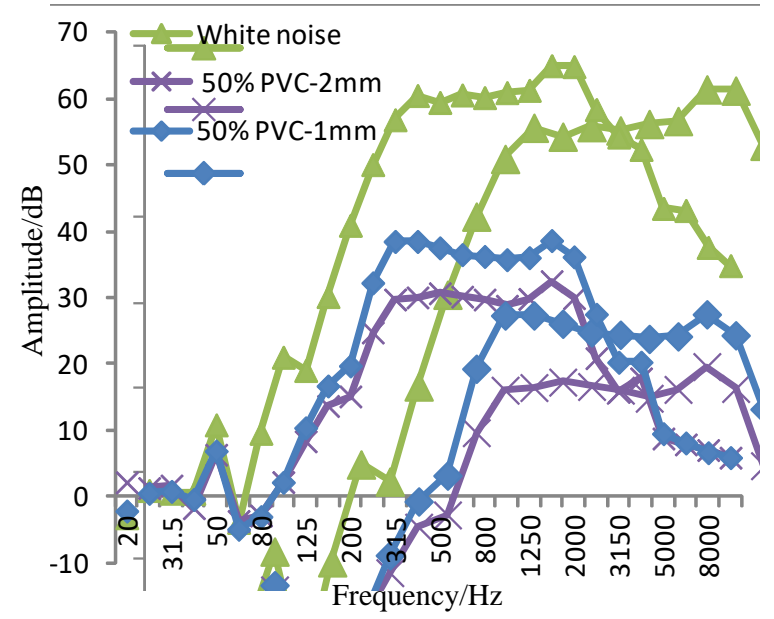

(a) White noise

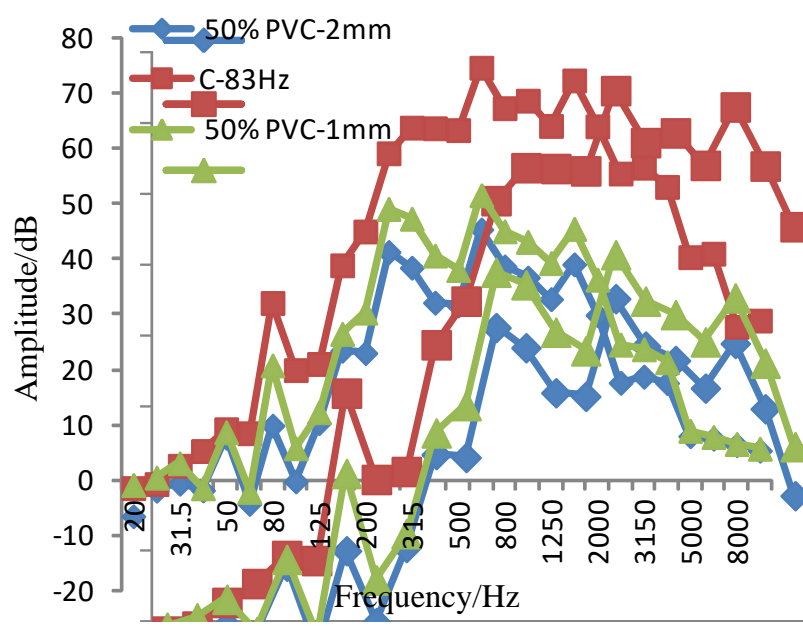

(b) Compressor noise

Fig.2. Noise reduction curves of PVC

From Fig. 2 we can see that amplitudes of low frequency were almost the same, however, amplitudes of medium-high frequency were greatly decreased after two types of noise are insulated by PVC. These curves show that sound insulation frequency of PVC is wide and noise reduction scope is mainly concentrated above $200 \mathrm{~Hz}$. Noise reduction effect is much better for medium-high frequency noise above $300 \mathrm{~Hz}$, especially for white noise amplitudes is reduced by half because the energy density is the same in white noise and easy to be isolated. Compressor noise has obvious order noise and frequency range with high energy is wide so the amplitudes after reduced of some frequency is higher than white noise. So insulation effect of PVC is not ideal for compressor noise and in practice PVC is used to reduce the compressor noise together with sound-absorbing materials. 


\section{Influence of areal density on PVC acoustic insulation}

Acoustic insulation cotton of PVC is sheet structure and acoustic insulation is influenced by many factors such as areal density, stiffness, material damping and boundary conditions [8]. Acoustic insulation is mainly reflected by the areal density of materials. From Noise reduction curves in Fig.2, we can see that acoustic insulation volume is little and sound waves have strong penetrability when frequency is small and acoustic insulation of PVC is influenced by its stiffness and damping. Above $125 \mathrm{~Hz}$, the acoustic insulation of PVC becomes big with frequency increasing. In the high frequency range, acoustic insulation volume rapidly increase and the noise reduction increase with frequency higher. For example, for white noise acoustic insulation volume increase from $24.4 \mathrm{~dB}$ at $250 \mathrm{~Hz}$ to $31.3 \mathrm{~dB}$ at $8000 \mathrm{~Hz}$. It is shown in Fig.2 that acoustic insulation of PVC with $2 \mathrm{~mm}$ is bigger than that of $1 \mathrm{~mm}$ in thickness and the maximum difference of acoustic insulation is $7 \mathrm{~dB}$. The test also show the areal density of $2 \mathrm{~mm}$ is about twice of $1 \mathrm{~mm}$ in thickness and for PVC the areal density become bigger with thickness increasing. So noise reduction effect is better as the areal density is bigger, which is mainly effected by the areal density. Therefore the PVC with bigger thickness should be used to effectively reduce noise in practice.

\section{Effect of $\mathrm{CaCO}_{3}$ on PVC acoustic insulation}

The acoustic insulation volume can be calculated as:

$$
N_{\mathrm{R}}=L_{1}-L_{2}
$$

(1)

In the type (1): $L_{1}$ is the source noise; $L_{2}$ is the noise after insulation. The acoustic insulation volume was compared of different $\mathrm{CaCO}_{3}$ content for two types of noise, see Fig.3. From Fig.3 we can see the acoustic insulation volume is all above $20 \mathrm{~dB}$, which show PVC is a better acoustic insulation material with $\mathrm{CaCO}_{3}$ content between $45 \%$ and $60 \%$. The acoustic insulation volume for the white noise is bigger than that for compressor noise because white noise has the same energy density but compressor noise has order noise.

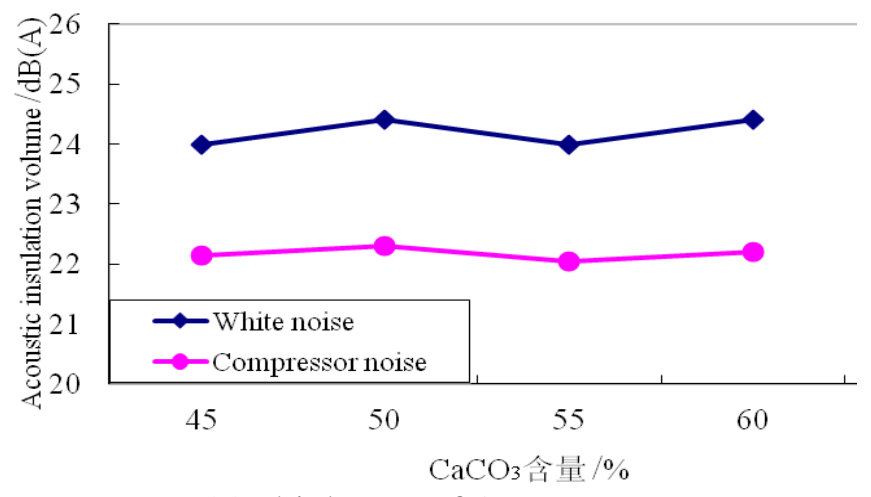

(a)Thickness of $1 \mathrm{~mm}$

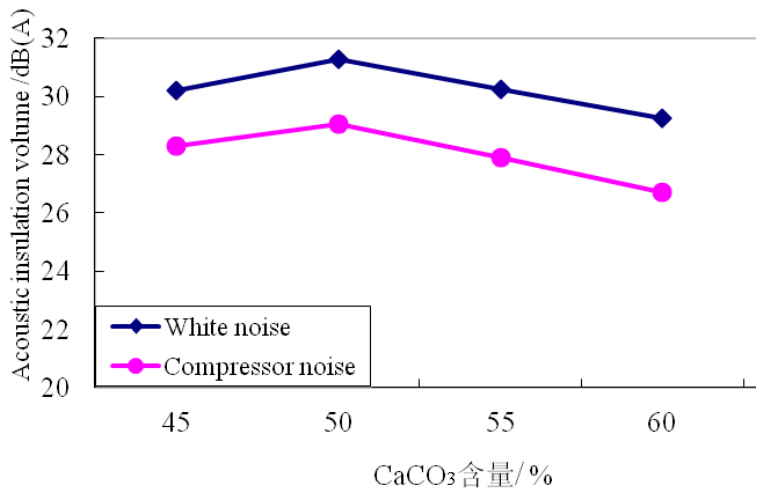

(b) Thickness of $2 \mathrm{~mm}$

Fig.3. Acoustic insulation of PVC of different thickness and content of $\mathrm{CaCO}_{3}$

Acoustic insulation of PVC is almost same with the increase of $\mathrm{CaCO}_{3}$ content in thickness of $1 \mathrm{~mm}$. And the sound insulation of PVC increases first and then decreases with the increase of $\mathrm{CaCO}_{3}$ content. So there is an optimum value soundproofing when the content of $\mathrm{CaCO}_{3}$ is $50 \%$. When the density of $\mathrm{CaCO}_{3}$ is very low in PVC which is no help for sound insulation. As the density of $\mathrm{CaCO}_{3}$ increase with the content of $\mathrm{CaCO}_{3}$ increasing bond strength between $\mathrm{CaCO}_{3}$ particles and PVC is high which will increase the number of sports in PVC matrix unit. So sonic in the material need to overcome greater resistance and then sound insulation is improved [6]. The sound insulation volume is max until content of $\mathrm{CaCO}_{3}$ is up to $50 \%$. However, if $\mathrm{CaCO}_{3}$ content continues to increase it cannot penetrate into the basis material PVC. So the composition of mixture material will maintain itself characteristics, thereby the amount of noise gradually reduce.

Overall difference is less than $2 \mathrm{~dB}$ between max and min insulation of PVC with different content of $\mathrm{CaCO}_{3}$. So the difference can be ignored when PVC is used to reduce noise together with 
sound-absorbing material in the application. The cost of PVC increase with content of $\mathrm{CaCO}_{3}$ decrease so PVC should be chosen as an insulation material with lower cost. But $\mathrm{CaCO}_{3}$ content must is less than $60 \%$, otherwise it can't meet the requirements of the mechanical properties.

\section{Conclusion}

The anechoic frequency band of PVC is wide and suitable to reduce medium-high frequency noise. Test results show that the acoustic insulation volume of PVC is higher than $20 \mathrm{~dB}$ and increases with the areal density increasing, which meet the requirement of acoustic material in air conditioner. Based on the test dates conclusion can be obtained that the acoustic insulation of PVC is best when $\mathrm{CaCO}_{3}$ content is $50 \%$, but in general the content of $\mathrm{CaCO}_{3}$ has little effect on the sound insulation of PVC. So PVC can be used to reduce compressor noise very well together with sound-absorbing material.

\section{Acknowledgements}

In this paper, the research was sponsored by the National Natural Science Foundation of China (Project NO.51405083) and the Project of Education Department of Guangdong (Project NO. 2014KQNCX186) and the School-level Training Plan for Excellent Young Teachers (Project NO. fsyq201409).

\section{References}

[1] Leli Cheng, Yuefei Yao, Xiaojie Tang etc. On the Flame-Retardant Properties of Soft PVC-Base Composite Insulation [J]. Journal of Zhejiang Technology University, 2011，28(2):160-165.

[2] Tianbing Yang, Yaqin FU. Sound insulation of honeycomb weave fabric reinforced polyvinylchloride composite [J]. Journal of Textile Research, 2011, 32(6):65-70.

[3] Hui Liu. Study on performance impact of filler to PVC flexible insulation composite material [D]. Zhejiang Technology University, 2012.

[4] Zhongcheng Fang, Mingbo Jiang, Yahu Zhang ect. Experimental research on PVC sound insulation improving the front noise of refrigerator [C]. Home appliance technology, 2014 (supplement):2-4. [5] Handong Xue, Xin Lai, Shiqiang Zhang etc. Research on Deadening Felt of Inverter Compressor [J]. Electrical Appliances, 2014(8):76-78.

[6] Lei Huang, Bowen Xia, Lianghong Tan etc. Noise Test and Control for Commercial Air Conditioner’s Compressors [J]. Noise and Vibration Control, 2015(1):191-194.

[7] Liping Zhang. Performances of PVC as Acoustical Insulating Material for the Air-conditioner [J]. Materials for Mechanical Engineering, Accept.

[8] Zhonglin Pan, Guoqing Zhai. Noise Control Technology [M].Chemical Industry Press.2006.4 